\title{
New Therapeutic Horizons for Graves' Hyperthyroidism
}

\author{
Laura C. Lane, ${ }^{1,2,3}$ Tim D. Cheetham, ${ }^{1,3}$ Petros Perros, ${ }^{2}$ and \\ Simon H. S. Pearce ${ }^{1,2}$
}

${ }^{1}$ Translational and Clinical Research Institute, Newcastle University, Newcastle-upon-Tyne NE1 3BZ, UK; ${ }^{2}$ Endocrine unit, Royal Victoria Infirmary, Newcastle-upon-Tyne Hospitals NHS Foundation Trust, Newcastle-upon-Tyne NE1 4LP, UK; and ${ }^{3}$ Department of Paediatric Endocrinology, The Great North Children's Hospital, Newcastle-upon-Tyne NE1 4LP, UK

ORCiD number: 0000-0002-6630-2701 (L. C. Lane); 0000-0001-8384-8063 (S. H. S. Pearce).

Abbreviations: ANCA, anti-neutrophil cytoplasmic antibody;BAFF, B-cell activating factor;BAFF-R, B-cell activating
factor receptor;cAMP, 3',5'-cyclic adenosine monophosphate;FcRn, neonatal immunoglobulin Fc receptor; G0, Graves'
orbitopathy;ITP, immune thrombocytopenia;MG, myasthenia gravis;RTX, rituximab;SLE, systemic lupus erythematosus;TNF,
tumor necrosis factor; TRAb, thyroid-stimulating hormone receptor autoantibody;TSH, thyrotropin (thyroid-stimulating
hormone); TSHR, thyroid-stimulating hormone receptor
Received: 17 June 2020; Accepted: 20 August 2020; First Published Online: 26 August 2020; Corrected andTypeset: 16 October 2020.

\section{Abstract}

Graves' hyperthyroidism is characterized by the presence of autoantibodies that stimulate the thyroid-stimulating hormone receptor (TSHR), resulting in uncontrolled secretion of excessive thyroid hormone. Conventional treatments, including antithyroid medication, radioiodine, or surgery have remained largely unchanged for the past 70 years and either lack efficacy for many patients, or result in lifelong thyroid hormone replacement therapy, in the case of the latter 2 options. The demand for new therapeutic options, combined with greater insight into basic immunobiology, has led to the emergence of novel approaches to treat Graves' hyperthyroidism. The current therapies under investigation include biologics, small molecules, and peptide immunomodulation. There is a growing focus on TSHR-specific treatment modalities, which carry the advantage of eliciting a specific, targeted approach, with the aim of avoiding disruption of the functioning immune system. These therapies present a new opportunity to supersede the inadequate treatments currently available for some Graves' patients, offering hope of successful restoration of euthyroidism without the need for ongoing therapy. Several of these therapeutic options have the potential to translate into clinical practice in the near future. This review provides a comprehensive summary of the recent advances and various stages of development of the novel therapeutic approaches to treat Graves' hyperthyroidism.

KeyWords: Graves' disease, hyperthyroidism, thyroid hormone, thyroid-stimulating hormone receptor, immunomodulation, immunotherapy 


\section{Graphical Abstract}

New therapeutic horizons for Graves' hyperthyroidism

Rituximab (anti-CD20)
Iscalimab (anti-CD40)
Belimumab (anti-BAFF)
Neonatal Fc receptor blockade
K1-70 (Blocking TRAb)
Small molecular TSHR antagonists
Immunomodulatory TSHR peptides $\longrightarrow$ immunomodulation

\section{ESSENTIAL POINTS}

- The conventional therapeutic options for Graves' disease have not improved over the past 70 years, despite substantial unmet clinical need and a significant lack of efficacy for many patients.

- The demand for innovative therapeutic options has led to the emergence of novel approaches in the treatment of Graves' hyperthyroidism, including biologic, small molecule, and peptide immunomodulation, several of which have translational potential into clinical practice in the near future.

- Targeting the direct cause of hyperthyroidism in Graves' disease using TSHR-specific modalities, has a clear advantage in providing a targeted approach while avoiding disruption of a functioning immune system.

- The introduction of novel therapeutics may lead to the restoration of a euthyroid state without the requirement for ongoing therapy, but the potential risk of immunocompromise and cost implications needs careful consideration.

The therapeutic options available for patients with Graves' hyperthyroidism have remained largely unchanged for the past 70 years, despite the current treatments having either limited efficacy or significant drawbacks. The classical approach to treating Graves' hyperthyroidism involves the administration of antithyroid drugs to block thyroid hormone synthesis, or alternatively, destruction or removal of the thyroid by radioiodine or surgery (1). Unfortunately, relapse occurs in over half of adults after stopping antithyroid drugs, and the lack of functional thyroid tissue following definitive treatment with radioiodine or surgery results in hypothyroidism with a lifelong requirement for levothyroxine replacement and associated clinical and biochemical monitoring. Patients with active Graves' orbitopathy (GO) are frequently managed with high-dose glucocorticoids, a treatment that has also been the mainstay of therapy for more than 50 years (2). Unfortunately, significant patient dissatisfaction with outcome and a reduced quality of life remains a concern despite improved surgical rehabilitation (3), and advances in this field are the subject of a recent review (4). This review summarizes the emergence of novel targeted therapies that are paving the way for a new era in the treatment of Graves' hyperthyroidism.

\section{Pathophysiology}

Graves' hyperthyroidism is an autoimmune condition that arises as a result of the loss of immunological tolerance to the thyroid-stimulating hormone receptor (TSHR) (1). Elevated circulating thyroid hormones in Graves' hyperthyroidism arise because of stimulating TSHR autoantibodies (TRAbs), which bind to leucine-rich repeats in the extracellular domain of the TSHR located on the surface of the thyrocytes (5). This mimics the action of thyroidstimulating hormone (TSH; thyrotropin) resulting in excessive, autonomous thyroid hormone production and hyperplasia of thyroid epithelial cells. The mature TSHR is a G protein-coupled receptor found primarily on thyroid follicular cells. It comprises an entirely extracellular A-subunit, and a B-subunit consisting of a small extracellular leader, 7 transmembrane domains, and an intracellular tail (5). The carboxy-terminal part of the A-subunit participates in a flexible hinge region; TSH binding to the leucine-rich repeats of the A-subunit results in a conformational change transduced through this hinge region, leading to a pivot or toggle of the 7 transmembrane domains. Part of this hinge region may be viewed as an autoligand or internal agonist which is displaced in response to both TSH and TRAb binding. Following the structural change in the transmembrane domains, Gs $\alpha$ signaling to increase $3^{\prime}, 5^{\prime}$-cyclic adenosine monophosphate (cAMP) and Gq signaling to activate phospholipase pathways, results in thyroid hormone synthesis and secretion (5). Interestingly, certain TSHR mutations may selectively abrogate Gq signaling without affecting the GSa-cAMP signal (6). It is 
known that TSHRs exist as homomultimeric complexes in certain situations (7), but whether this is influenced by ligand binding, or is necessary for signaling is under active investigation.

Histologically, the thyroid gland in Graves' hyperthyroidism demonstrates diffuse follicular cell hyperplasia with varying extents of lymphocytic infiltration into the thyroid stroma (8). These infiltrates consist of both T- and B lymphocytes and may form ectopic intrathyroidal germinal centers, creating an important source of the pathogenic TRAb-producing plasma cells (9). Extrathyroidal TRAb production has been found at regional bone marrow and lymph nodes sites (10), explaining why TRAbs, albeit at reduced concentrations, persist following surgical removal of the thyroid gland (11).

The TSHR is also found in orbital fibroblasts and the upper dermis where binding of TRAbs results in a proliferative response that contributes to the extrathyroidal signs seen in Graves' hyperthyroidism, GO, and pretibial myxedema (5). Clinical manifestations of GO affect about $25 \%$ of people diagnosed with Graves' hyperthyroidism, and it can be facially disfiguring, may cause functional visual disabilities, and carries the potential for occasional loss of sight (12).

Although the specific immune mechanisms driving Graves' hyperthyroidism remain unknown, a greater understanding of the interactions between $\mathrm{B}$ and $\mathrm{T}$ lymphocytes and the autoantigen-antibody complex has enabled progressive insights into the immunopathology underpinning Graves' hyperthyroidism (5). The role of B cells in Graves' hyperthyroidism is well established, not only in their capacity as immunoglobulin-secreting plasma cells producing the pathogenic TRAb autoantibody, but also in their ability to act as antigen-presenting cells, likely presenting TSHR epitopes to T cells to perpetuate disease, as well as modulating the immune response by producing both pro- and anti-inflammatory cytokines and chemokines (13).

The described immunological insights have led to the emergence of several novel therapeutic options, most of which remain under investigation in clinical or preclinical studies (Table 1). In addition, Graves' hyperthyroidism, in contrast to many other autoimmune conditions, has a specific autoantigen which is the extracellular domain of the TSHR. Thus, the TSHR provides an ideal therapeutic candidate for targeted immunomodulation. Current therapeutic options for the treatment of Graves' hyperthyroidism that are discussed below include those directly targeting the $\mathrm{B}$ cells or their associated interactors and cytokines, or alternatively, specific TSHR modulation by the use of small molecule antagonists, antagonistic TSHR monoclonal antibodies, or "tolerogenic" TSHR peptides (Fig. 1). The advantages and disadvantages of the current and novel therapeutic options for Graves' hyperthyroidism are described in Table 2.

Table 1. Summary of the Novel Therapeutic Approaches Being Investigated and Their Potential or Proven Efficacy in the Treatment of Graves' Hyperthyroidism

\begin{tabular}{|c|c|c|c|c|}
\hline Mechanism & Novel therapies & Stage of development & $\begin{array}{c}\text { Potential efficacy } \\
\text { in GH }\end{array}$ & $\begin{array}{l}\text { Proven efficacy } \\
\text { in GH }\end{array}$ \\
\hline B-cell depletion & $\begin{array}{l}\text { Rituximab }(A n t i-C D 20 \\
\quad m A b)\end{array}$ & Phase 2 trials $(14,15)$ & + & $+(*)$ \\
\hline $\begin{array}{l}\text { Blocking CD40 receptor interactions (blocks } \\
\text { CD40-CD154 co-stimulatory pathway, } \\
\text { attenuating B cell activation) }\end{array}$ & $\begin{array}{l}\text { Iscalimab (CFZ533) } \\
\qquad(A n t i-C D 40 m A b)\end{array}$ & Phase 2 trial (16) & +++ & $++(*)$ \\
\hline $\begin{array}{l}\text { Blocking } \mathrm{FcRn}-\operatorname{IgG} \text { interactions (inhibits } \operatorname{Ig} G \\
\text { recycling) }\end{array}$ & $\begin{array}{l}\text { RVT-1401 } \\
\text { Rozanolixizumab } \\
\text { Efgartigimod }\end{array}$ & $\begin{array}{l}\text { Phase } 2 \text { trial (RVT-1401, } \\
\text { NCT03922321) }\end{array}$ & +++ & ND \\
\hline $\begin{array}{l}\text { Blocking BAFF interaction (reduces B cell } \\
\quad \text { proliferation and survival) }\end{array}$ & $\begin{array}{l}\text { Belimumab }(\text { Anti-BAFF } \\
\quad m A b)\end{array}$ & $\begin{array}{c}\text { Phase } 2 \text { trial (EudraCT } \\
\text { 2015-002127-26) }\end{array}$ & ++ & ND \\
\hline $\begin{array}{l}\text { Small molecule TSHR antagonists (directly } \\
\quad \text { inhibit TSHR signaling) }\end{array}$ & ANTAG-3 VA-K-14 S37a & Preclinical $(17-20)$ & +++ & ND \\
\hline $\begin{array}{l}\text { TSHR-blocking antibodies (blocks TSHR } \\
\text { stimulation by TSH or TRAbs) }\end{array}$ & K1-70 (Anti-TSHR $m A b)$ & $\begin{array}{l}\text { Case report (21) Phase } 1 \text { trial } \\
\quad(\text { NCT02904330) }\end{array}$ & +++ & ++ \\
\hline $\begin{array}{l}\text { TSHR-specific immunotherapy (induces } \\
\text { tolerogenic immune response) }\end{array}$ & $\begin{array}{l}\text { ATX-GD-59 (TSHR } \\
\text { peptide) }\end{array}$ & Phase I trial (22) & +++ & $++(*)$ \\
\hline
\end{tabular}

,,++++++ ; strength of potential or proven efficacy based on current available evidence

Abbreviations: BAFF, B-cell activating factor; FcRn, neonatal immunoglobulin Fc receptor; GH, Graves' hyperthyroidism; mAb, monoclonal antibody; ND, no data; TSHR, thyroid-stimulating hormone receptor; TRAbs; TSH receptor autoantibodies.

(*) in those with low pretreatment TRAb levels 


\section{Novel Therapeutic Strategies}

\section{B-lymphocyte depletion (CD20 depletion)}

As a humorally driven condition, the essential role of $\mathrm{B}$ cells in Graves' hyperthyroidism provides a logical therapeutic target for immunomodulatory treatment. The B-cell depleting therapy rituximab (RTX) has been used for more than 20 years to treat lymphoproliferative malignancies, such as lymphoma, with increasing use over the past decade to treat autoimmune disease. Although the anti-CD20 monoclonal antibody RTX is the most widely studied of the B-cell therapies, the precise mechanisms by which RTX produces a beneficial effect remain uncertain. Direct apoptosis, complement-mediated cytotoxicity, and antibody-dependent cellular cytotoxicity all appear to contribute to RTX efficacy in depleting B cells (23). The CD20 transmembrane protein is expressed on $\mathrm{B}-$ lineage lymphocytes, from early pre-B to mature and memory B cells; however, CD20 expression is lost during $\mathrm{B}$-cell differentiation into mature antibody-secreting plasma cells. Circulating B-lymphocyte depletion is very rapid, occurring within a few hours of RTX infusion. The survival of long-lived plasma cells (CD20-negative) and refractory memory $\mathrm{B}$ cells in lymphoid tissues explains how RTX-treated individuals are still able to mount an immune response against naturally encountered and previously vaccinated pathogens (24). Although the reasons remain unclear, memory $B$ cells appear to be more resistant to depletion with RTX compared to naïve B cells, allowing them to be recruited in a secondary immune response (25).

RTX was originally reported to have efficacy in controlling rheumatoid arthritis, with subsequent studies in myasthenia gravis (MG), anti-neutrophil cytoplasmic antibody (ANCA)-associated vasculitis, systemic lupus erythematosus (SLE) and multiple sclerosis (26-30). Several groups have investigated the use of RTX in Graves' hyperthyroidism. Fassi et al undertook a prospective study of 20 patients with Graves' hyperthyroidism comparing short-term treatment using methimazole with or without RTX. Although they demonstrated some efficacy with sustained remission in the RTX group (4/10 patients) after a mean of 23 months follow-up, it appeared most effective in those with low TRAb levels at presentation (<5 IU/L) (14). Another small prospective study by Heemstra et al, including 13 patients with recurrent Graves' hyperthyroidism, demonstrated that $70 \%$ remained euthyroid after RTX treatment with significantly decreased TRAb and free thyroxine levels, after a mean follow-up duration of 18 months (15). Similarly, the patients who responded had low pretreatment TRAb levels (median 4 IU/L) and less severe hyperthyroidism.

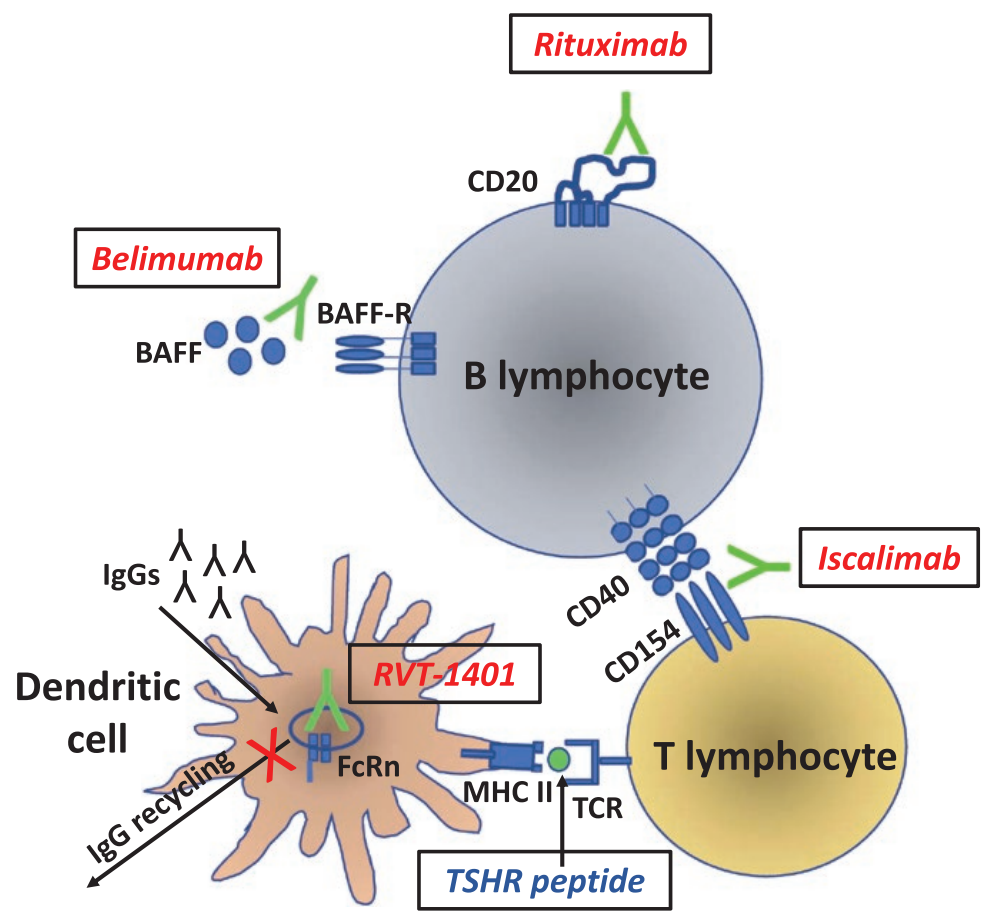

TSH Receptor

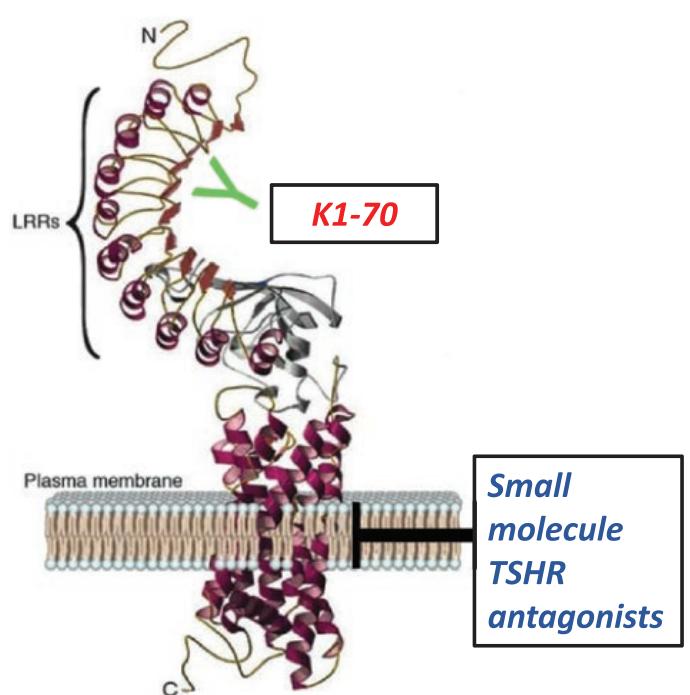

Figure 1. Illustration of novel therapeutic approaches in the treatment of Graves' hyperthyroidism. The therapeutics in red indicate those that are monoclonal antibodies. Abbreviations: BAFF, B-cell activating factor; BAFF-R, B cell activating factor receptor; FcRn, neonatal immunoglobulin Fc receptor; IgGs, immunoglobulins; K1-70,TSHR-blocking antibody; MHC II, major histocompatibility complex class II; RVT-1401, FcRn blocker;TCR, T cell receptor; TSHR, thyroid-stimulating hormone receptor. TSH receptor image reprinted by permission from Springer Nature: Immunologic Research. Morshed SA, Latif R, Davies TF. Delineating the autoimmune mechanisms in Graves' disease. 2012. 
Table 2. Advantages and Disadvantages of Therapeutic Options in Graves' Hyperthyroidism

\begin{tabular}{|c|c|c|}
\hline Therapy & Advantages & Disadvantages \\
\hline \multirow[t]{3}{*}{ Antithyroid drugs (CBZ, PTU, MMI) } & Noninvasive, oral tablet & High risk of recurrence \\
\hline & Outpatient therapy & Frequent monitoring/clinic attendance \\
\hline & No radiation hazard or surgical risk & $\begin{array}{c}\text { Risk of minor (eg, rash, urticaria, arthralgia) or major side } \\
\text { effects (eg, agranulocytosis, hepatotoxicity, vasculitis) }\end{array}$ \\
\hline \multirow[t]{5}{*}{ Radioiodine } & Definitive treatment & Radiation exposure \\
\hline & Outpatient procedure & Risk of exacerbation/development of GO \\
\hline & Few adverse effects & Permanent hypothyroidism \\
\hline & Cost effective & Need to delay pregnancy and avoid breastfeeding \\
\hline & Reduces goiter size & Radiation safety precautions \\
\hline \multirow[t]{6}{*}{ Thyroidectomy } & Definitive treatment & Inpatient procedure \\
\hline & Effective & Requires anesthetic \\
\hline & & Permanent hypothyroidism \\
\hline & & Surgical complications (RLN damage, HPT) \\
\hline & & Scarring \\
\hline & & Postoperative pain \\
\hline \multirow[t]{7}{*}{ B-cell therapies } & $\begin{array}{l}\text { *Avoids side effects associated with } \\
\text { ATD }\end{array}$ & $*$ Cost implications \\
\hline & $\begin{array}{l}\text { *Potential for restoration of } \\
\text { euthyroidism without ongoing } \\
\text { therapy }\end{array}$ & $\begin{array}{l}* * \text { Uncertain effect on long-term hypothyroidism, goiter size, } \\
\text { and prevention of relapse }\end{array}$ \\
\hline & May benefit GO & Infusion side effects \\
\hline & & Risk of infection \\
\hline & & Most effective in those with low TRAb levels \\
\hline & & Potential thromboembolic events (iscalimab) \\
\hline & & Increased psychiatric events (belimumab) \\
\hline \multirow[t]{7}{*}{ TSHR-targeted therapies } & As above* and: & As above $* *$ \\
\hline & No global immunosuppression & \\
\hline & May benefit GO & \\
\hline & Small molecule TSHR antagonists: & \\
\hline & May be active in oral form & \\
\hline & May be effective independent of & \\
\hline & TRAb concentration & \\
\hline \multirow[t]{3}{*}{ TSHR peptide desensitization } & As above* and: & As above** \\
\hline & No global immunosuppression & Potential disease exacerbation in susceptible individuals \\
\hline & May benefit GO & Given subcutaneously (bruising/swelling at injection site) \\
\hline
\end{tabular}

Abbreviations: ATD, antithyroid drug; CBZ, carbimazole; GO, Graves’ orbitopathy; HPT, hypoparathyroidism; MMI, methimazole; PTU, propylthiouracil; RLN, recurrent laryngeal nerve; TRAb, thyroid-stimulating hormone receptor autoantibodies.

Some, but not all, studies have shown a reduction in serum TRAb antibody levels following RTX therapy (14, $15,31)$, but the reduction does not correlate with the extent of B-cell depletion achieved $(15,32)$, nor does the effect seem to be greater than that expected with antithyroid medication alone (14). Additionally, in contrast to the previous prospective studies specifically investigating the effect of RTX on hyperthyroidism, several studies, including 2 RCTs, have examined the effect of RTX on GO $(33,34)$. Although studies show a mixed outcome $(4,33,34)$, the clinical improvement of GO seen with RTX appears to occur independently of thyroid function or circulating thyroid antibodies $(15,31,32,35-37)$.
Most patients will have complete ( $>99 \%$ ) B-cell depletion following a single $500 \mathrm{mg}$ dose of RTX, and this persists for 5 to 20 months before recovery of the peripheral B-cell compartment (38), with recent evidence suggesting that much lower doses of RTX $(100 \mathrm{mg})$ are equally effective (39). The vast majority of residual peripheral B cells exhibit a plasma cell or memory cell phenotype (38); however, complete intrathyroidal B cell depletion has been demonstrated following RTX therapy (40). The persistence of residual autoreactive memory B cells alongside plasma cells, which may continue to produce the pathogenic TRAb autoantibody, may contribute to the lack of definitive success in treating Graves' hyperthyroidism with 
RTX. A detailed assessment of the immunological response in patients with type 1 diabetes suggests that RTX does not reset early B-cell tolerance checkpoints and that this may be why durable remission does not occur (41). The results of a clinical trial investigating RTX therapy in 27 young (12-20 years of age) patients with Graves' hyperthyroidism are awaited (ISRCTN20381716; the RIGD study); however, current evidence does not support the routine use of RTX in Graves' hyperthyroidism in adults.

Adverse effects have been reported with RTX, the most frequent of which is a mild infusion reaction including throat itching and nasal congestion, which resolves on slowing the infusion with or without the administration of hydrocortisone. There have also been reports of articular and gastrointestinal symptoms, specifically colitis, related to circulating immune complexes following RTX (42). The rare complication of progressive multi-focal leukoencephalopathy has largely been reported in patients with predisposing comorbidities who have received multiple immunosuppressive agents (43). An increased risk of serious infection has been reported in RTX-treated patients, but this tends to occur in patients with concomitant severe immunodeficiency or those with underlying malignancy (44). RTX has been used for many years in the treatment of various autoimmune diseases, and a review over 9.5 years involving repeated courses of RTX in rheumatoid arthritis patients demonstrated no evidence of an increased safety risk or increased reporting rates of any type of adverse events compared with placebo plus methotrexate (45). In addition, the vast majority of studies using RTX in Graves' hyperthyroidism or GO have demonstrated no serious adverse events $(15,31-33,36,37)$. Newer second-generation CD20-depleting strategies, including ocrelizumab and ofatumumab, which theoretically have lower immunogenicity and improved tolerability $(46,47)$, may provide a future option for Graves' patients.

\section{Disruption of B-cell activation or activity}

\section{Blocking CD40 interactions}

CD40, a tumor necrosis factor (TNF) family receptor found on thyrocytes and antigen-presenting cells, including B cells, has a primary role in coordinating effective antigen presentation (48). Its ligand CD154 (CD40 ligand; CD40L) is transiently expressed on activated $\mathrm{T}$ cells and other nonimmune cells under inflammatory conditions. The CD40-CD154 interaction initiates a co-stimulatory pathway that provides the second signal required for the initiation of an adaptive humoral immune response (1). This interaction between $\mathrm{B}$ and $\mathrm{T}$ lymphocytes is proposed to have a central role in the pathogenesis of Graves' hyperthyroidism as it is required for intrathyroidal germinal center formation, and for maturing the B-cell repertoire to allow production of pathogenic thyroid-stimulating antibodies $(1,9,49)$.

Variants in the CD40 gene have been associated with several autoimmune diseases, including Graves' hyperthyroidism, where they appear to influence thyroid antibody production and provide a predictive marker of relapse (50-53). Functional studies have demonstrated that the diseaseassociated variant in CD40 alters the consensus Kozak initiation sequence, resulting in increased translational efficiency and suggesting that overexpression of CD40 has a causative association with the predisposition to Graves' hyperthyroidism (54). Indeed, several different murine models have confirmed that either genetic or chemical modulation of CD40 signaling can modify the severity of autoimmune thyroiditis or thyroid autoantibody production $(49,55,56)$, establishing CD40 as a potential therapeutic target in the treatment of Graves' hyperthyroidism.

The anti-CD40 monoclonal antibody iscalimab (CFZ533) targets the CD40-CD154 co-stimulatory pathway, resulting in attenuation of the B-cell activation signal (57). Iscalimab is a nondepleting (Fc silent) antibody, designed to block the $\mathrm{CD} 40$ receptor interactions without the removal of CD40-expressing cells. Of the autoimmune conditions, it has been best studied in Sjögren's syndrome, where an RCT demonstrated that iscalimab was welltolerated and led to clinical improvement (58).

In a recent open-label study, 15 adult patients with untreated Graves' hyperthyroidism were given 5 doses of intravenous iscalimab over a 12 -week period (16). During the 24-week follow-up period, 7 (47\%) patients were deemed "responders" with normal free triiodothyronine and free thyroxine levels and without a need for additional antithyroid medication during this period. In addition, TRAb concentrations were significantly reduced following iscalimab treatment, with $4(27 \%)$ patients achieving normal TRAb levels by week 20, all of whom were "responders." Those that did not respond were noted to have higher baseline TRAb levels, to have a larger goiter, and to be more likely to use cigarettes and have GO. CD40 engagement by iscalimab decreased total cell-surface CD40 protein on peripheral B cells by $40 \%$, persisting for at least 8 weeks after the last dose was administered. Levels of serum CXCL13, a chemokine with an essential role in germinal center activity, also significantly declined following treatment (16). The decreased CXCL13 concentrations may inhibit the formation of ectopic intrathyroidal germinal centers in Graves' hyperthyroidism. Indeed, CD40 blockade has been demonstrated to inhibit ectopic lymphoid structures in inflamed tissue in the murine model of Sjögren's syndrome (59).

Iscalimab was found to be safe and well-tolerated, with no serious treatment-related adverse events reported. 
Similar to RTX, iscalimab is an immunosuppressive therapy and therefore risk of infection is always a concern. CD40 is expressed on vascular endothelium and platelets, so thromboembolic complications have been highlighted as another hypothetical risk-although this was not observed in the Graves' disease patients treated. Unfortunately, after the 24-week follow-up period, 4 of the 7 "responders" relapsed and 3 of these patients required low-dose antithyroid medication.

Targeted blockade of the CD40-CD154 interaction shows promising results in Graves' hyperthyroidism, at least in the short-term and in those with lower TRAb levels (<20 IU/L). The fact that CD40 receptors are also present on orbital fibroblasts suggests the potential for a beneficial effect on GO as well. Indeed, 2 patients with GO whose hyperthyroidism responded to iscalimab also had improvement of eye symptoms and signs (16). An RCT of longer duration to assess the possibility of sustained remission is now warranted.

\section{Blocking immunoglobulin recycling (FcRn therapeutics)}

The long half-life associated with IgG antibodies, such as TRAbs, is attributed to the neonatal immunoglobulin Fc receptor $(\mathrm{FcRn})$, which recycles endocytosed $\mathrm{IgG}$ antibody by binding it in the acidic conditions of the lysosome and recycling it to the cell membrane for release back into the circulation (60). FcRn-deficient mice have shown resistance to autoimmune disease (61), and blockade of FcRn has resulted in the amelioration of autoimmune disease in different animal models $(62,63)$. Therefore, inhibition of FcRn is an attractive novel therapeutic concept for IgG-mediated autoimmune diseases like Graves' hyperthyroidism, where acclerated antibody catabolism and the associated reduction in the levels of circulating pathogenic TRAb would be benefical.

The 2 most widely studied compounds that target FcRn are efgartigimod and rozanolixizumab (64), both of which are currently in phase 3 studies for autoimmune disease. Efgartigimod is a human $\operatorname{IgG}_{1}$-derived $\mathrm{Fc}$ fragment, while rozanolixizumab is a humanized, $\mathrm{IgG}_{4}$ anti-FcRn monoclonal antibody, both of which block FcRn-IgG interactions, thereby inhibiting $\operatorname{IgG}$ recycling and accelerating the removal of pathogenic IgG autoantibodies from the circulation $(60,65)$. The therapeutic effect of intravenous immunoglobins in several autoimmune diseases is also mediated by functional Fc receptor blockade, but these novel FcRn therapeutics demonstrate increased receptor binding affinity, which results in efficacy at a much lower dose (less than $50 \mathrm{mg} / \mathrm{kg}$ vs $1-2 \mathrm{~g} / \mathrm{kg}$ body weight) $(66,67)$. Both efgartigimod and rozanolixizumab caused a sustained reduction in circulating $\mathrm{IgG}$ levels of approximately $75 \%$ to $90 \%$, both in preclinical studies of murine models of autoimmune disease (arthritis and encephalitis) $(63,68)$ and in healthy human subjects $(64,69)$.

Efgartigimod is currently being investigated in MG, immune thrombocytopenia (ITP), and pemphigus vulgaris. In a phase 2 RCT of efgartigimod in 12 patients with generalized MG, a rapid decrease of up to $70 \%$ in total $\operatorname{IgG}$ and anti-acetylcholine receptor autoantibody levels was demonstrated, alongside a rapid and sustained clinical improvement in $75 \%$ of those treated, compared with $25 \%$ of the placebo group (70). Similar findings were demonstrated in a phase II trial in ITP with a reduction in total IgG level associated with significantly increased platelet count and reduced bleeding (71). Rozanolixizumab has also undergone phase 2 trials in patients with ITP and MG, demonstrating almost a $70 \%$ mean reduction of serum $\mathrm{IgG}$ and $\operatorname{IgG}$ autoantibodies associated with clinical response (72). These studies have demonstrated both efgartigimod and rozanolixizumab to be well-tolerated, with a mild headache being the most frequently described side effect and no treatment-related serious or severe adverse events reported (70-72).

Other potential Fc and Fc $\gamma$ R-targeting therapeutics for autoimmune disease are in various phases of development from preclinical to phase 2 studies (64). Although neither rozanolixizumab nor efgartigimod have been investigated in Graves' hyperthyroidism, a phase 2 trial with another biologic FcRn blocker, RVT-1401, is about to report (NCT03922321) and it seems likely that these approaches are worthy of further exploration in Graves' hyperthyroidism.

\section{Inhibition of B-cell proliferation and differentiation (blockade of B-cell activating factor)}

B-cell activating factor (BAFF) is a member of the TNF family of cytokines and has an essential role in B-lymphocyte activation, differentiation, and survival. Elevated circulating BAFF levels have been found in patients with several autoimmune conditions, including active Graves' hyperthyroidism, where both degree of elevation of thyroid hormones and TRAb concentrations have been demonstrated to correlate with serum BAFF levels (73). Genetic variants in BAFF have also been associated with susceptibility to Graves' hyperthyroidism $(74,75)$. Therefore, BAFF is a logical therapeutic target molecule for B-cell-driven autoimmune conditions such as Graves' hyperthyroidism.

The BAFF monoclonal antibody belimumab binds to and antagonizes the biological activity of soluble BAFF. Blocking the interaction of BAFF with its receptor negatively effects B-cell proliferation, indirectly decreasing B-cell survival and reducing production of autoantibodies 
(76). The increased thyroidal expression of BAFF and its primary receptor, BAFF-R, in the infiltrating immune cells and thyrocytes in patients with Graves' hyperthyroidism suggests a central role of the BAFF-BAFF-R interactions in its pathogenesis (77). With this rationale, belimumab treatment is currently under investigation in an RCT conducted in Graves' hyperthyroidism with active orbitopathy (EudraCT 2015-002127-26).

Efficacy of belimumab alone, or in combination with RTX, has been demonstrated in SLE, with significant improvements in long-term organ damage $(78,79)$. Conversely, belimumab was not found to be efficacious in patients with ANCA-associated vasculitis (80). Belimumab is currently licensed for treatment of seropositive SLE and, although it has been found generally safe and well-tolerated with no increased risk of serious infection $(78,80)$, an increased risk of psychiatric events has been reported by the UK Medicines and Healthcare products Regulatory Agency in patients with SLE on belimumab.

As discussed above, the isolated use of a B-cell depleting therapy (eg, RTX) to treat Graves' hyperthyroidism may lack efficacy due to the persistence of long-lived plasma cells and residual memory cells. Furthermore, following B-cell depletion there is a surge in plasma BAFF concentration. Concurrent targeting of both the homeostatic factors that are responsible for B-cell proliferation and differentiation, along with a B-cell depleting therapy may enhance the effect on tissue-resident autoreactive B cells.

\section{TSHR-specific modalities}

As the direct cause of hyperthyroidism in Graves' disease is stimulation of the TSHR, several groups have been developing approaches that directly prevent TSHR signaling, either through small molecules or by using antibodies that block receptor activation. Furthermore, TSHR peptides are being investigated to see if they may have long-lasting immunomodulatory properties. A key advantage of this strategy when compared to the immunomodulatory strategies described above is their more specific, targeted approach which-from a theoretical standpoint—is unlikely to have a deleterious impact on the patient's ability to fight infection.

\section{Small molecule TSHR antagonists}

Small molecule agonists and antagonists have the potential to directly stimulate or inhibit TSHR signaling that could lead to highly potent therapies for thyroid dysfunction. A series of compounds that inhibit TSHR function as inverse agonists (inhibiting basal as well as agonist-induced signaling) have been developed; the best studied compound has been termed ANTAG-3 $(17,18)$. These compounds inhibit TSH-stimulated cAMP production in vitro and lower thyroid hormone levels in mice treated with the thyroidstimulating monoclonal antibody M22, suggesting likely efficacy in inhibiting TRAb-induced Graves' hyperthyroidism. Two other TSHR antagonist compounds, VA-K-14 and S37a, have been identified by high-throughput library screening and both are able to inhibit TSH- and patient TRAb-induced signaling in vitro $(19,20)$. A key issue is the structural homology between the TSHR, and the follicle stimulating hormone receptor and lutenizing hormone/chorionic gonadotropin receptors, which gives rise to the potential for off-target reproductive effects. VA-K-14 gives minor antagonism of FSH signaling in vitro, whereas S37a appears to be more specific. Elegant structural studies have shown that S37a binds TSHR in the juxta-membrane region between the C-terminal end of the extracellular domain, which has an internal agonist function, and the first extracellular loop (81). VA-K-14 and ANTAG3 most likely fit within a hydrophobic pocket that more directly stabilizes the transmembrane domains in an "off" conformation $(17,19)$. This modeling suggests that all 3 of these compounds interact with TSHR at sites that are distinct from the extracellular domain site of both TSH and TRAb binding, meaning that they should be effective irrespective of circulating TRAb concentration. These small molecule compounds are expected to be active orally, and because of their precise targeting to the TSHR they are anticipated to be suitable for long-term administration. None of these compounds have been trialed in humans yet, but this approach may hold promise for both long-term control of hyperthyroidism without the need for thyroid ablation and for the amelioration of thyroid eye disease.

\section{TSHR-blocking antibodies}

Occasional patients with GO may present with active inflammatory eye disease in the presence of hypothyroidism and high titers of TRAb antibodies, which contain a proportion of TSHR-blocking antibodies. Sanders et al were able to clone a monoclonal TSHR-blocking antibody, termed K1-70 from such a patient (82). In rats, K1-70 was able to completely suppress the serum thyroxine rise following administration of the stimulating M22 antibody, suggesting potential efficacy in Graves' hyperthyroidism with high serum TRAb levels (83). K1-70 has been given on compassionate grounds to a patient whose metastatic follicular thyroid cancer was being driven by TRAb antibodies associated with co-existing GO (21). The response in terms of thyroid-stimulating activity in the serum was dramatic; cancer progression was initially arrested, and eye disease improved markedly and in a sustained fashion. Thus, this treatment clearly holds promise for both Graves' hyperthyroidism and GO. There is an ongoing phase 1 trial (NCT02904330) of K1-70 in treatment-naïve Graves' patients, which is due to report in the next year. 
TSHR-specific immunotherapy

Conventional approaches to combating autoimmune disease involve using drugs with broad immunosuppressive effects and hence the potential side effect of susceptibility to infection. An alternative approach that has been successfully used to treat allergic disease is to desensitize the immune response to the specific allergen (immune stimulant) (84). The principle that administering small and increasing doses of soluble antigen can lead to tolerogenic immune responses has now also been demonstrated in several autoimmune diseases, leading to a new therapeutic approach (85). However, there is a concern that administration of an antigen in a susceptible individual could lead to exacerbation of disease, as seen in some preclinical settings (86). Repeated administration of tolerogenic peptides from the insulin molecule has been successful in reducing $\mathrm{HbA} 1 \mathrm{c}$ and insulin requirements in newly diagnosed adults with type 1 diabetes (87). In a similar approach, Jansson et al identified dominant TSHR T-cell epitopes. These were administered to HLA-DR3 transgenic mice that had been primed for hyperthyroidism by TSHR cDNA immunization, and the TSHR peptide-pretreated mice showed a profound reduction in induced TRAbs and lower thyroid hormone levels (88). In a phase 1 study, this TSHR peptide mixture, termed ATX-GD-59, was then administered every 2 weeks subcutaneously to patients with treatmentnaïve Graves' hyperthyroidism, leading to normalization of hyperthyroidism in 5 of 10 patients and improvement in 7 of 10 (22). TRAb levels improved in line with reducing thyroid hormone levels. This novel treatment may have very little in the way of side effects except for swelling and bruising at the injection site. Phase 2 studies are awaited.

\section{Limitations}

The described novel therapeutic approaches are not without potential limitations. As well as cost implications and the potential risk of immunocompromise with the non-TSHRspecific therapies, it remains unclear whether they will ameliorate the long-term risk of hypothyroidism, reduce goiter, or indeed prevent a late relapse of Graves' hyperthyroidism. It seems likely that patients with Graves' hyperthyroidism will continue to be at risk for thyroid dysfunction after cessation of therapy, necessitating annual biochemical monitoring.

\section{Conclusion}

Conventional treatment of Graves' hyperthyroidism with surgery, radioiodine, or antithyroid drugs has not substantially changed since the late 1940s. An abundance of new therapeutic approaches, involving biologic, small molecule, and peptide immunomodulation are currently at different stages of development and several will translate into the clinic over the next few years. These therapies may render destructive radioiodine thyroid ablation and thyroidectomy obsolete treatments of historical interest, although the advantages of restoring a euthyroid state without the need for ongoing therapy will need to be balanced against potential risks such as immunocompromise. Future studies need to focus on understanding the most effective combinations of the conventional medical and surgical therapies with these recently introduced options.

\section{Acknowledgments}

Financial Support: This work was supported by the Medical Research Council (MRC): Grant number MR/S001611/1.

\section{Additional Information}

Correspondence: Dr Laura Lane, Translational and Clinical Research Institute, Central Parkway, Newcastle-upon-Tyne, NE1 3BZ. E-mail: Laura.Lane@newcastle.ac.uk.

Disclosure Summary: S.H.S.P. was chief investigator of the phase 1 trial of immunomodulatory TSHR peptide therapy. S.H.S.P. has received speaker fees from Quidel, Sanofi, Berlin Chemie. P.P. was a collaborator/subinvestigator for the peptide immunotherapy study.

Data Availability: Data sharing is not applicable to this article as no datasets were generated or analyzed during the current study.

\section{References}

1. Smith TJ, Hegedüs L. Graves' disease. N Engl J Med. 2016;375(16):1552-1565.

2. Genere N, Stan MN. Current and emerging treatment strategies for Graves' orbitopathy. Drugs. 2019;79(2):109-124.

3. Ponto KA, Binder H, Diana T, et al. Prevalence, phenotype, and psychosocial well-being in euthyroid/hypothyroid thyroidassociated orbitopathy. Thyroid. 2015;25(8):942-948.

4. Graves' orbitopathy review due to be published in Endocrine Reviews (editorial team to add).

5. Morshed SA, Latif R, Davies TF. Delineating the autoimmune mechanisms in Graves' disease. Immunol Res. 2012;54(1-3):191-203.

6. Kleinau G, Worth CL, Kreuchwig A, et al. Structural-functional features of the thyrotropin receptor: a class a g-protein-coupled receptor at work. Front Endocrinol (Lausanne). 2017;8:86.

7. Rapoport B, Aliesky HA, Chen CR, McLachlan SM. Evidence that TSH receptor A-subunit multimers, not monomers, drive antibody affinity maturation in graves' disease. J Clin Endocrinol Metab. 2015;100(6):E871-E875.

8. LiVolsi VA, Baloch ZW. The pathology of hyperthyroidism. Front Endocrinol (Lausanne). 2018;9:737.

9. Armengol MP, Juan M, Lucas-Martín A, et al. Thyroid autoimmune disease: demonstration of thyroid antigen-specific B cells and recombination-activating gene expression in chemokinecontaining active intrathyroidal germinal centers. Am J Pathol. 2001;159(3):861-873. 
10. Weetman AP, McGregor AM, Wheeler MH, Hall R. Extrathyroidal sites of autoantibody synthesis in Graves' disease. Clin Exp Immunol. 1984;56(2):330-336.

11. Kautbally S, Alexopoulou O, Daumerie C, Jamar F, Mourad M, Maiter D. Greater efficacy of total thyroidectomy versus radioiodine therapy on hyperthyroidism and thyroidstimulating immunoglobulin levels in patients with Graves' disease previously treated with antithyroid drugs. Eur Thyroid J. 2012;1(2):122-128.

12. Tanda ML, Piantanida E, Liparulo L, et al. Prevalence and natural history of Graves' orbitopathy in a large series of patients with newly diagnosed graves' hyperthyroidism seen at a single center. J Clin Endocrinol Metab. 2013;98(4):1443-1449.

13. Shen P, Fillatreau S. Antibody-independent functions of B cells: a focus on cytokines. Nat Rev Immunol. 2015;15(7):441-451.

14. El Fassi D, Nielsen CH, Bonnema SJ, Hasselbalch HC, Hegedüs L. B lymphocyte depletion with the monoclonal antibody rituximab in Graves' disease: a controlled pilot study. $J$ Clin Endocrinol Metab. 2007;92(5):1769-1772.

15. Heemstra KA, Toes RE, Sepers J, et al. Rituximab in relapsing Graves' disease, a phase II study. Eur J Endocrinol. 2008;159(5):609-615.

16. Kahaly GJ, Stan MN, Frommer L, et al. A novel anti-CD40 monoclonal antibody, iscalimab, for control of graves hyperthyroidism-a proof-of-concept trial. J Clin Endocrinol Metab. 2020;105(3):dgz013.

17. Neumann S, Huang W, Eliseeva E, Titus S, Thomas CJ, Gershengorn MC. A small molecule inverse agonist for the human thyroid-stimulating hormone receptor. Endocrinology. 2010;151(7):3454-3459.

18. Neumann S, Nir EA, Eliseeva E, et al. A selective TSH receptor antagonist inhibits stimulation of thyroid function in female mice. Endocrinology. 2014;155(1):310-314.

19. Latif R, Realubit RB, Karan C, Mezei M, Davies TF. TSH receptor signaling abrogation by a novel small molecule. Front Endocrinol (Lausanne). 2016;7:130.

20. Marcinkowski P, Hoyer I, Specker E, et al. A new highly thyrotropin receptor-selective small-molecule antagonist with potential for the treatment of Graves' orbitopathy. Thyroid. 2019;29(1):111-123.

21. Furmaniak J, Ryder M, Castro M, et al. Blocking the TSH receptor with the human monoclonal autoantibody K1-70(TM) improves Graves' ophthalmopathy and aids control of advanced follicular thyroid carcinoma-results of long-term treatment under the first in human single patient expanded use therapy. Eur Thyroid J. 2018;7(suppl 1):Abstract P22.

22. Pearce SHS, Dayan C, Wraith DC, et al. Antigen-specific immunotherapy with thyrotropin receptor peptides in Graves' hyperthyroidism: a phase I study. Thyroid. 2019;29(7):1003-1011.

23. Pavanello F, Zucca E, Ghielmini M. Rituximab: 13 open questions after 20years of clinical use. Cancer Treat Rev. 2017;53:38-46.

24. Cho A, Bradley B, Kauffman R, et al. Robust memory responses against influenza vaccination in pemphigus patients previously treated with rituximab. JCI Insight. 2017;2(12):e93222.

25. Leandro MJ. B-cell subpopulations in humans and their differential susceptibility to depletionwith anti-CD20 monoclonal antibodies. Arthritis Res Ther. 2013;15(Suppl 1):S3.
26. Edwards JC, Szczepanski L, Szechinski J, et al. Efficacy of B-celltargeted therapy with rituximab in patients with rheumatoid arthritis. N Engl J Med. 2004;350(25):2572-2581.

27. Hehir MK, Hobson-Webb LD, Benatar M, et al. Rituximab as treatment for anti-MuSK myasthenia gravis: Multicenter blinded prospective review. Neurology. 2017;89(10):1069-1077.

28. Specks U, Merkel PA, Seo P, et al.; RAVE-ITN Research Group. Efficacy of remission-induction regimens for ANCA-associated vasculitis. N Engl J Med. 2013;369(5):417-427.

29. Leandro MJ, Cambridge G, Edwards JC, Ehrenstein MR, Isenberg DA. B-cell depletion in the treatment of patients with systemic lupus erythematosus: a longitudinal analysis of $24 \mathrm{pa}-$ tients. Rheumatology (Oxford). 2005;44(12):1542-1545.

30. Hauser SL, Waubant E, Arnold DL, et al.; HERMES Trial Group. B-cell depletion with rituximab in relapsing-remitting multiple sclerosis. N Engl J Med. 2008;358(7):676-688.

31. Mitchell AL, Gan EH, Morris M, et al. The effect of B cell depletion therapy on anti-TSH receptor antibodies and clinical outcome in glucocorticoid-refractory Graves' orbitopathy. Clin Endocrinol (Oxf). 2013;79(3):437-442.

32. Salvi M, Vannucchi G, Campi I, et al. Treatment of Graves' disease and associated ophthalmopathy with the anti-CD20 monoclonal antibody rituximab: an open study. Eur J Endocrinol. 2007;156(1):33-40.

33. Salvi M, Vannucchi G, Currò N, et al. Efficacy of B-cell targeted therapy with rituximab in patients with active moderate to severe Graves' orbitopathy: a randomized controlled study. J Clin Endocrinol Metab. 2015;100(2):422-431.

34. Stan MN, Garrity JA, Carranza Leon BG, Prabin T, Bradley EA, Bahn RS. Randomized controlled trial of rituximab in patients with Graves' orbitopathy. J Clin Endocrinol Metab. 2015;100(2):432-441.

35. Salvi M, Vannucchi G, Campi I, et al. Efficacy of rituximab treatment for thyroid-associated ophthalmopathy as a result of intraorbital B-cell depletion in one patient unresponsive to steroid immunosuppression. Eur J Endocrinol. 2006;154(4):511-517.

36. Silkiss RZ, Reier A, Coleman M, Lauer SA. Rituximab for thyroid eye disease. Ophthalmic Plast Reconstr Surg. 2010;26(5):310-314.

37. Vannucchi G, Campi I, Bonomi M, et al. Rituximab treatment in patients with active Graves' orbitopathy: effects on proinflammatory and humoral immune reactions. Clin Exp Immunol. 2010;161(3):436-443.

38. Leandro MJ, Cambridge G, Ehrenstein MR, Edwards JC. Reconstitution of peripheral blood B cells after depletion with rituximab in patients with rheumatoid arthritis. Arthritis Rheum. 2006;54(2):613-620.

39. Salvi M, Vannucchi G, Currò N, et al. Small dose of rituximab for graves orbitopathy: new insights into the mechanism of action. Arch Ophthalmol. 2012;130(1):122-124.

40. El Fassi D, Clemmensen O, Nielsen CH, Silkiss RZ, Hegedüs L. Evidence of intrathyroidal B-lymphocyte depletion after rituximab therapy in a patient with Graves' disease. J Clin Endocrinol Metab. 2007;92(10):3762-3763.

41. Chamberlain N, Massad C, Oe T, Cantaert T, Herold KC, Meffre E. Rituximab does not reset defective early B cell tolerance checkpoints. J Clin Invest. 2016;126(1):282-287. 
42. El Fassi D, Nielsen CH, Junker P, Hasselbalch HC, Hegedüs L. Systemic adverse events following rituximab therapy in patients with Graves' disease. J Endocrinol Invest. 2011;34(7):e163-e167.

43. Berger JR, Malik V, Lacey S, Brunetta P, Lehane PB. Progressive multifocal leukoencephalopathy in rituximab-treated rheumatic diseases: a rare event. J Neurovirol. 2018;24(3):323-331.

44. Gea-Banacloche JC. Rituximab-associated infections. Semin Hematol. 2010;47(2):187-198.

45. van Vollenhoven RF, Emery P, Bingham CO $3^{\text {rd }}$, et al. Longterm safety of rituximab in rheumatoid arthritis: 9.5-year follow-up of the global clinical trial programme with a focus on adverse events of interest in RA patients. Ann Rheum Dis. 2013;72(9):1496-1502.

46. Du FH, Mills EA, Mao-Draayer Y. Next-generation anti-CD20 monoclonal antibodies in autoimmune disease treatment. Auto Immun Highlights. 2017;8(1):12.

47. Bar-Or A, Grove RA, Austin DJ, et al. Subcutaneous ofatumumab in patients with relapsing-remitting multiple sclerosis: the MIRROR study. Neurology. 2018;90(20):e1805-e1814.

48. Lee HJ, Lombardi A, Stefan M, et al. CD40 signaling in Graves disease is mediated through canonical and noncanonical thyroidal nuclear factor $\kappa B$ activation. Endocrinology. 2017;158(2):410-418.

49. Huber AK, Finkelman FD, Li CW, et al. Genetically driven target tissue overexpression of CD40: a novel mechanism in autoimmune disease. J Immunol. 2012;189(6):3043-3053.

50. Jacobson EM, Huber AK, Akeno N, et al. A CD40 Kozak sequence polymorphism and susceptibility to antibody-mediated autoimmune conditions: the role of CD40 tissue-specific expression. Genes Immun. 2007;8(3):205-214.

51. Tomer Y, Concepcion E, Greenberg DA. A C/T single-nucleotide polymorphism in the region of the CD40 gene is associated with Graves' disease. Thyroid. 2002;12(12):1129-1135.

52. Ban Y, Tozaki T, Taniyama M, Tomita M, Ban Y. Association of a C/T single-nucleotide polymorphism in the 5' untranslated region of the CD40 gene with Graves' disease in Japanese. Thyroid. 2006;16(5):443-446.

53. Wang PW, Chen IY, Juo SH, Hsi E, Liu RT, Hsieh CJ. Genotype and phenotype predictors of relapse of graves' disease after antithyroid drug withdrawal. Eur Thyroid J. 2013;1(4):251-258.

54. Jacobson EM, Concepcion E, Oashi T, Tomer Y. A Graves' disease-associated Kozak sequence single-nucleotide polymorphism enhances the efficiency of CD40 gene translation: a case for translational pathophysiology. Endocrinology. 2005;146(6):2684-2691.

55. Chen CR, Aliesky HA, Guo J, Rapoport B, McLachlan SM. Blockade of costimulation between $\mathrm{T}$ cells and antigenpresenting cells: an approach to suppress murine Graves' disease induced using thyrotropin receptor-expressing adenovirus. Thyroid. 2006;16(5):427-434.

56. Carayanniotis G, Masters SR, Noelle RJ. Suppression of murine thyroiditis via blockade of the CD40-CD40L interaction. Immunology. 1997;90(3):421-426.

57. Ristov J, Espie P, Ulrich P, et al. Characterization of the in vitro and in vivo properties of CFZ533, a blocking and nondepleting anti-CD40 monoclonal antibody. Am J Transplant. 2018;18(12):2895-2904.

58. Farag A, Papas A, Fisher B, et al. Novel anti-CD40 monoclonal antibody CFZ533 in patients with primary sjogren syndrome: a phase iia double-blind, placebo controlled randomized trial. Oral Surg Oral Med Oral Pathol. 2018;126(4):e203-e204.

59. Wieczorek G, Bigaud M, Pfister S, et al. Blockade of CD40-CD154 pathway interactions suppresses ectopic lymphoid structures and inhibits pathology in the NOD/ShiLtJ mouse model of Sjögren's syndrome. Ann Rheum Dis. 2019;78(7):974-978.

60. Smith B, Kiessling A, Lledo-Garcia R, et al. Generation and characterization of a high affinity anti-human FcRn antibody, rozanolixizumab, and the effects of different molecular formats on the reduction of plasma IgG concentration. Mabs. 2018;10(7):1111-1130.

61. Li N, Zhao M, Hilario-Vargas J, et al. Complete FcRn dependence for intravenous Ig therapy in autoimmune skin blistering diseases. J Clin Invest. 2005;115(12):3440-3450.

62. Liu L, Garcia AM, Santoro H, et al. Amelioration of experimental autoimmune myasthenia gravis in rats by neonatal FcR blockade. J Immunol. 2007;178(8):5390-5398.

63. Patel DA, Puig-Canto A, Challa DK, Perez Montoyo H, Ober RJ, Ward ES. Neonatal Fc receptor blockade by Fc engineering ameliorates arthritis in a murine model. J Immunol. 2011;187(2):1015-1022.

64. Zuercher AW, Spirig R, Baz Morelli A, Rowe T, Käsermann F. Next-generation $F_{C}$ receptor-targeting biologics for autoimmune diseases. Autoimmun Rev. 2019;18(10):102366.

65. Kiessling P, Lledo-Garcia R, Watanabe S, et al. The FcRn inhibitor rozanolixizumab reduces human serum IgG concentration: a randomized phase 1 study. Sci Transl Med. 2017;9(414):eaan1208. doi:10.1126/scitranslmed.aan1208

66. Yu Z, Lennon VA. Mechanism of intravenous immune globulin therapy in antibody-mediated autoimmune diseases. $N$ Engl J Med. 1999;340(3):227-228.

67. Vaccaro C, Zhou J, Ober RJ, Ward ES. Engineering the Fc region of immunoglobulin $\mathrm{G}$ to modulate in vivo antibody levels. Nat Biotechnol. 2005;23(10):1283-1288.

68. Challa DK, Bussmeyer U, Khan T, et al. Autoantibody depletion ameliorates disease in murine experimental autoimmune encephalomyelitis. Mabs. 2013;5(5):655-659.

69. Ulrichts P, Guglietta A, Dreier T, et al. Neonatal Fc receptor antagonist efgartigimod safely and sustainably reduces IgGs in humans. J Clin Invest. 2018;128(10):4372-4386.

70. Howard JF Jr, Bril V, Burns TM, et al.; Efgartigimod MG Study Group. Randomized phase 2 study of FcRn antagonist efgartigimod in generalized myasthenia gravis. Neurology. 2019;92(23):e2661-e2673.

71. Newland AC, Sánchez-González B, Rejtô L, et al. Phase 2 study of efgartigimod, a novel FcRn antagonist, in adult patients with primary immune thrombocytopenia. Am J Hematol. 2020;95(2):178-187.

72. Robak T, Kazmierczak M, Jarque I, et al. Safety and efficacy of an anti-fcrn antibody, rozanolixizumab, in patients with primary immune thrombocytopenia: interim results of a phase II, multipledose study: S850. HemaSphere. 2019;3:379 (Abstract S850).

73. Lin JD, Wang YH, Fang WF, et al. Serum BAFF and thyroid autoantibodies in autoimmune thyroid disease. Clin Chim Acta. 2016;462:96-102.

74. Lane LC, Allinson KR, Campbell K, et al. Analysis of BAFF gene polymorphisms in UK Graves' disease patients. Clin Endocrinol (Oxf). 2019;90(1):170-174. 
75. Lin JD, Yang SF, Wang YH, et al. Analysis of associations of human BAFF gene polymorphisms with autoimmune thyroid diseases. Plos One. 2016;11(5):e0154436.

76. Stohl W, Hiepe F, Latinis KM, et al.; BLISS-52 Study Group; BLISS-76 Study Group. Belimumab reduces autoantibodies, normalizes low complement levels, and reduces select B cell populations in patients with systemic lupus erythematosus. Arthritis Rheum. 2012;64(7):2328-2337.

77. Campi I, Tosi D, Rossi S, et al. B cell activating factor (BAFF) and BAFF receptor expression in autoimmune and nonautoimmune thyroid diseases. Thyroid. 2015;25(9):1043-1049.

78. Navarra SV, Guzmán RM, Gallacher AE, et al.; BLISS-52 Study Group. Efficacy and safety of belimumab in patients with active systemic lupus erythematosus: a randomised, placebo-controlled, phase 3 trial. Lancet. 2011;377(9767): 721-731.

79. Kraaij T, Kamerling SWA, de Rooij ENM, et al. The NET-effect of combining rituximab with belimumab in severe systemic lupus erythematosus. J Autoimmun. 2018;91:45-54.

80. Jayne D, Blockmans D, Luqmani R, et al.; BREVAS Study Collaborators. Efficacy and safety of belimumab and azathioprine for maintenance of remission in antineutrophil cytoplasmic antibody-associated vasculitis: a randomized controlled study. Arthritis Rheumatol. 2019;71(6):952-963.

81. Marcinkowski P, Kreuchwig A, Mendieta S, et al. Thyrotropin receptor: allosteric modulators illuminate intramolecular signaling mechanisms at the interface of ecto- and transmembrane domain. Mol Pharmacol. 2019;96(4): 452-462.

82. Evans M, Sanders J, Tagami T, et al. Monoclonal autoantibodies to the TSH receptor, one with stimulating activity and one with blocking activity, obtained from the same blood sample. Clin Endocrinol (Oxf). 2010;73(3):404-412.

83. Furmaniak J, Sanders J, Young S, et al. In vivo effects of a human thyroid-stimulating monoclonal autoantibody (M22) and a human thyroid-blocking autoantibody (K1-70). Auto Immun Highlights. 2012;3(1):19-25.

84. Akdis CA, Akdis M. Mechanisms of allergen-specific immunotherapy and immune tolerance to allergens. World Allergy Organ J. 2015;8(1):17.

85. Streeter HB, Rigden R, Martin KF, Scolding NJ, Wraith DC. Preclinical development and first-in-human study of ATX-MS-1467 for immunotherapy of MS. Neurol Neuroimmunol Neuroinflamm. 2015;2(3):e93.

86. Genain CP, Abel K, Belmar N, et al. Late complications of immune deviation therapy in a nonhuman primate. Science. 1996;274(5295):2054-2057.

87. Alhadj Ali M, Liu YF, Arif S, et al. Metabolic and immune effects of immunotherapy with proinsulin peptide in human newonset type 1 diabetes. Sci Transl Med. 2017;9(402).

88. Jansson L, Vrolix K, Jahraus A, Martin KF, Wraith DC. Immunotherapy with apitopes blocks the immune response to TSH receptor in HLA-DR transgenic mice. Endocrinology. 2018;159(9):3446-3457. 